\title{
Making the World a Better Place: Sharing Experiences at Malaysian Field Hospital, Bangladesh via Photos and Written Communication
}

\author{
$\underline{\text { Huzairi }}{ }^{1}$, Nada S.Z ${ }^{2}$, Khalilah A.B ${ }^{3}$, Shamsul B ${ }^{3}$ \\ ${ }^{1}$ Universiti Teknologi MARA, Medical Faculty, UiTM Sungai Buloh, Selangor, Malaysia; ${ }^{2}$ Pathology \\ Department, Penang General Hospital, Ministryof Health, Malaysia, ${ }^{3}$ Malaysian Field Hospital, Ukhia, \\ Bangladesh
}

ABSTRACT

Introduction: Malaysian Field Hospital (MFH) in the district of Ukhia, Bangladesh has been operating since December 2017. It is an effort by the Malaysian government to assist in the humanitarian crisis involving Rohingya refugees in Bangladesh.

Objective: To share experiences in the tertiary care services provided by MFH.

Methods: Visits to the MFH and refugee camps were made by volunteers. With consent, personal interviews with staff and patients were conducted and recorded on paper or by photography.

Results: MFH provides specialist care in general surgery, internal medicine, anaesthesiology, obstetrics \& gynaecology, intensive care as well as general in-patient and outpatient services. It also provides $\mathrm{X}$-ray imaging, transfusion, dental, pharmacist, logistic, administrative and utensil sterilization services. MFH acts as a referral centre for 286 primary health clinics and secondary hospitals including the Turkish Field Hospital, Medicins Sans Frontieres (MSF), International Federation of Red Cross and Red Crescent Societies (IFRC) and HOPE hospital. Up to $31^{\text {st }}$ December 2018, MFH has treated 50,588 patients and conducted 1,268 surgical procedures. In partnership with the World Health Organization (WHO) and United Nations High Commission for Refugees (UNHCR), MFH has been involved in the strategic planning to ease refugee influx.

Conclusion: Information sharing through stories and photos depicting the actual situation in refugee camps should be facilitated to promote awareness and to positively alter the mass' attitude and perceptions whilst preserving confidentiality and dignity.

Keywords: Rohingya, refugee, Malaysia Field Hospital 